\title{
Correction to: Retrospectives: Magnetic Resonance Studies of Intrinsic Defects in Semiconductors
}

\author{
Correction to: \\ Chapter 3 in: P.G. Baranov et al., Magnetic Resonance \\ of Semiconductors and Their Nanostructures, \\ Springer Series in Materials Science 253, \\ https://doi.org/10.1007/978-3-7091-1157-4_3
}

The original version of chapter 3 was inadvertently published with incorrect cross citations of the references. The chapter has been modified now with correct references cross citations corresponding to the references list. 